\title{
Modelo de gestión logística para pequeñas y medianas empresas en México
}

\author{
Patricia Cano Olivos \\ Universidad Politécnica \\ de Tlaxcala \\ patricia.cano@uptlax.edu.mx
}

\section{Fernando Orue}

Carrasco

Universidad Popular Autónoma del Estado de Puebla

fernando.orue@upaep.mx

\section{José Luis Martínez}

Flores

Universidad Popular Autónoma

del Estado de Puebla

joseluis.martinez01@upaep.mx

\section{Yésica Mayett Moreno}

Popular Autónoma del Estado de Puebla

yesica.mayett@upaep.mx

Gabriel López Nava

Universidad Nacional Autónoma de México

glopez@bits-group.com

\section{Resumen}

La apertura de los mercados y la globalización de las cadenas de suministro demandan cambios estructurales en los que la logística juega un papel estratégico. Actualmente, los clientes evalúan la calidad del producto, el valor agregado del mismo y su disponibilidad en tiempo y forma, de ahí la necesidad de hacer eficientes los procesos. Diversos expertos han propuesto modelos de gestión logística para elevar la competitividad en el mercado; algunos de ellos son ambiciosos para las pequeñas y medianas empresas (Pyme) debido a la estructura informal y carencia de conocimientos técnicos de las mismas; otros hacen referencia indirecta a los flujos de información interna, implicando una desintegración total del sistema por la débil interrelación entre áreas. La Pyme en México representa el 4.2\% de las empresas, genera el $31.5 \%$ del empleo y aporta el $37 \%$ del Producto Interno Bruto; de ahí surge la importancia de fortalecer su posición competitiva en el mercado. Esta investigación presenta el diseño de un modelo conceptual de gestión logística para Pyme que podría dar solución integral a través del control de las variables involucradas en los procesos logísticos; para verificar que las variables consideradas en cada dimensión identificada son las correctas se utilizó el análisis factorial.

Palabras clave: modelos de gestión logística, Pyme, competitividad, cadena de suministro, análisis factorial 


\title{
Logistics management model for small and medium sized enterprises in Mexico
}

\begin{abstract}
The market opening and the globalization of supply chains, demand a structural changes in which logistics has a strategic role. Nowadays, clients evaluate product quality, its value added and its availability in time and form, which implies a need to make the process efficient. Several experts have proposed some logistics management models to increase competitiveness in the market, but some of these models are too ambitious for small and medium sized enterprises (SMEs) due to their informal structure and their lack of technical knowledge; other models make indirect reference to the internal information flows, which include whole system disintegration because of the weak interrelation among the different areas. In Mexico, SMEs represent $4.2 \%$, they generate $31.5 \%$ of employment and provide $37 \%$ of the gross domestic product; that is why it is important to reinforce their competitive position in the market. This research presents the design of a conceptual model of logistic management for SMEs which could provide comprehensive solution through control of the variables involved in the logistics process; to verify that the variables considered in each identified dimension are correct factor analysis was used.
\end{abstract}

Keywords: logistic management models, SMEs, competitiveness, supply chain, factor analysis

\section{Introducción}

La logística se encarga de la administración del flujo de materiales e información a lo largo del proceso de creación de valor: aprovisionamiento, producción y distribución. De esta manera, gestiona un grupo de actividades que tienen lugar en la organización con la finalidad de brindar valor al cliente mediante la transformación de los factores productivos (Ballou, 2004).

En la actualidad la globalización de los mercados hace más complejo el sistema logístico y su gestión; por ello, es necesario mejorar las condiciones de las compañías productoras o comercializadoras de bienes y servicios que se encuentran participando en un ambiente de negocios internacionales.

Ante las nuevas condiciones de alta competitividad, la adecuada gestión de la cadena de suministro y la logística juegan un papel muy importante, ya sea para las empresas que exportan o para las que producen para el mercado doméstico, sin importar si son pequeñas o grandes. Sin embargo, un modelo de gestión logística 
para la Pyme debe ser distinto al de la gran empresa, básicamente debido a los recursos tecnológicos utilizados, el lenguaje, la estructura y la cultura bajo la cual operan (Velásquez, 2003), a diferencia de los altos niveles de capacitación y recursos económicos con los que operan las grandes empresas.

La función logística por sí misma tiene una gran importancia, pero a su vez también genera un efecto de tracción del desarrollo del sector privado y del crecimiento en el resto de los actores económicos de un país o región. Una logística eficiente y accesible constituye un elemento clave para que las empresas, en particular la Pyme, puedan competir con éxito en el contexto de la globalización (Banco Interamericano de Desarrollo, 2011).

De acuerdo con el índice de desempeño logístico, publicado bianualmente por el Banco Mundial, de 155 países, México ocupó la posición 47 con una calificación global de 3.06 equivalente al 66\% (Banco Mundial, 2012), que comparado con el tamaño de su economía y actividad de comercio exterior se considera una calificación baja.

En México la Pyme representa el 4.2\% de las empresas del país, genera el 31.5\% del empleo, aporta cerca del $37.0 \%$ del producto Interno Bruto (PIB) y, además, constituye la columna vertebral de la economía nacional por los acuerdos comerciales que el país ha tenido en los ultimos años. Ante estas circunstancias, el Gobierno Federal de Mexico ha desarrollado una estrategia nacional que apoye a las empresas a elevar la competitividad de las mismas a través de talleres de benchmarking de procesos logísticos y de gestión de la cadena de suministro a nivel micro, pequeña y mediana empresa (Gobierno Federal, 2007).

Considerando este panorama, la búsqueda de soluciones alternas para fortalecer la Pyme se vuelve indispensable. La revisión de la literatura permitió identificar diversos modelos integrales de gestión logística para elevar la competitividad en el mercado; a partir de esta revisión, la mayoría de autores proponen modelos que resultan ambiciosos para una Pyme debido a su estructura informal y a la carencia de conocimientos técnicos. Otros autores y organismos han desarrollado modelos de gestión logística centrados en las características de la Pyme; sin embargo, aún quedan desatendidos los flujos de información internos necesarios para su adecuada implementación, debilitando la interrelación entre cada una de las áreas involucradas en la gestión, lo que propicia una desintegración del sistema como un todo. 
En la primera parte de este artículo se analiza la importancia de la gestión logística para mejorar la competitividad de la Pyme en México de manera general y las del sector de manufactura textil en particular, las cuales fueron elegidas para aplicar el instrumento de medición por su alta concentración en el estado de Tlaxcala (estado que representa un reto especial ante su actual pérdida de competitividad); además, se presenta la revisión de la literatura y la información de fuentes de datos secundarios que permitió identificar modelos de gestión logística para la Pyme. En la segunda parte se presenta el diseño de un modelo de gestión logística que pueda facilitar la administración integral de las cuatro áreas identificadas como las más importantes para este tipo de empresas, así como la aplicación de herramientas que pueden mejorar el desempeño logístico de su cadena de suministro. Finalmente, se verifican las variables consideradas en el modelo mediante el análisis estadístico multivariante, se presentan las conclusiones de la investigación y se discute la recomendación de diversas líneas para futuras investigaciones.

\section{Revisión de literatura}

En la revisión de la literatura fueron incluidos tres temas principales: a) índice de desempeño logístico, b) Pyme y c) modelos de gestión logística.

a) Índice de desempeño logístico. Éste es publicado cada dos años por el Banco Mundial; en su reporte de 2012 indica que México está situado en el lugar 47 de un total de 155 países encuestados con un desempeño global logístico del 3.06 equivalente al 66\%. Esta medición de desempeño consiste en una escala de 1 a 5, donde 1 es el nivel menos eficiente y 5 el nivel más eficiente. El desempeño logístico de México en los seis factores que mide el Banco Mundial fue: seguimiento y rastreo: 3.15 , aduanas: 2.63 , competencia y calidad logística: 3.02 , infraestructura: 3.03, tiempos de entrega: 3.47 y envíos internacionales: 3.07 . Estas mediciones y posiciones de México en el ranking mundial revelan que existen oportunidades de mejora en las diferentes áreas de la logística, mismas que de ser corregidas, permitirán elevar en alguna medida la competitividad del país.

b) Pyme. En el nivel internacional más del $90 \%$ de las empresas son micro, pequeñas y medianas; representan el sector de la economía que aporta el mayor número de unidades económicas y más del 50\% del empleo. En América Latina este estrato representa entre el 60 y 90\% de todas las unidades económicas (INEGI, 2011). 
A pesar de que la Pyme tiene importancia en el nivel internacional y nacional, en general, carece de una estructura formal en la mayoría de sus áreas (Díaz et al., 2012; Domínguez, 2010; Robles y Carlos, 2003). Debido a su tamaño y la escasez de recursos económicos es común que los empleados realicen varias actividades para muchas de las cuales no son especialistas; suele observarse que las mismas personas que dirigen sean quienes también ejecuten funciones de planeación financiera, producción, administración de personal y comercialización, entre otras. La falta de especialización los lleva a desarrollar actividades logísticas de bajo nivel debido a la falta de conocimientos técnicos y la aplicación incorrecta del concepto de cadena de suministro (BID, 2011). Las diversas crisis internas que esto genera repercute en su productividad y competitividad (Daft, 2011; Rodríguez, 2003), entendida esta última como la capacidad para atraer y retener inversiones y talento (Consejo Ejecutivo de Empresas Globales, 2004; Instituto Mexicano para la Competitividad, 2010), así como en la capacidad de mantener o incrementar la participación en el mercado con estrategias empresariales sin sacrificar utilidades (Hernández-Laos, 2000).

La logística permite a las empresas proyectar dichas actividades en un escenario externo, visualizar las actividades y enlaces necesarios para comercializar los productos y elevar su competitividad en los niveles nacional o internacional. Las actividades logísticas son el motor para nuevas inversiones en infraestructura, por lo que las plataformas logísticas integrales son una forma de enlazar la oferta y la demanda (Secretaría de Economía, 2011) en los niveles empresarial y nacional.

Uno de los grandes retos de la Pyme en nuestro país es la ausencia de formalización (Díaz et al., 2012); es decir, la falta de procedimientos, de una estructura organizada, de programas y de planificación. El desempeño económico de una empresa está directamente relacionado con su gestión y eficacia.

En las últimas cinco décadas, la industria textil ha enfrentado crisis recurrentes debido a la transición paulatina de una producción masiva estandarizada a otra orientada a la moda (Vera, 2010); el proceso de adaptación ha generado bajas en la producción, empleo y ventas, además de una continua pérdida de competitividad (Instituto Tecnológico Autónomo de México, 2010; Vera, 2010); por ello, será importante que las empresas de manufactura textil, particularmente la Pyme, se inserten en este proceso para poder mantenerse en el mercado. 
c) Modelos de gestión logística. Algunos expertos han propuesto modelos integrales de gestión logística para elevar la competividad en el mercado:

1. La Secretaría de Economía de México (2011) diseñó un modeloque considera 16 capacidades: estrategia y desempeño de la organización, servicio al cliente, gestión de procesos, administración de la demanda, abastecimiento y compras, procesamiento de órdenes de clientes, operación del almacén, administración del inventario, transporte, logística inversa, seguridad en el transporte de mercancías, procesos de importación/exportación, capacidad financiera, conciencia ambiental, sistemas de información y gestión de datos, organización, personal y competencias.

2. El modelo de referencia de operaciones de la cadena de suministro (SCORmodel:supply-chainoperations reference-model), desarrolladoporelSupply Chain Council of North America (1996), no tiene descripción matemática ni métodos heurísticos; es una herramienta estándar que analiza y mejora el desempeño de la cadena de suministro de las organizaciones usando KPI's (Key Performance Indicators). El SCOR identifica principalmente cinco procesos de gestión, tales como planificación, aprovisionamiento, manufactura, distribución/entrega y devolución.

Los modelos anteriores resultan muy ambiciosos aplicados al contexto de una Pyme porque han sido diseñados bajo el criterio de una empresa de estructura estable; la Secretaría de Economía de México propone un modelo de verificación de las capacidades logísticas de la empresas que serán evaluadas por un organismo certificador para poder otorgar el sello de confianza de los procesos logísticos; los indicadores a los que tienen que dar cumplimiento son poco alcanzables a corto plazo. Por otro lado, el SCOR-model no describe cada proceso de negocio o actividad (Calderón y Francisco, 2005), por lo que en la Pyme es muy difícil su implementación, pues en su mayoría la estructura de ésta es inestable y carece de conocimientos técnicos sólidos.

Otros autores y organismos han desarrollado modelos de gestión logística centrados en las características específicas de la Pyme, entre los que podemos mencionar los siguientes:

- Díaz et al. (2008) identificaron tres componentes principales en la cadena de suministro: provisión, producción y distribución. Aunque insinúan la 
integración de todos los participantes de la cadena de suministro, los autores sólo estudian las partes susceptibles de costeo dentro de ésta, dejando a un lado la importancia de la gestión para mejorar el desempeño logístico de la cadena de suministro.

- González et al. (2012) presentan una metodología de gestión logística para el mejoramiento de pequeñas empresas e identifican cinco áreas de oportunidad: aprovisionamiento, almacenamiento, distribución, costos y servicio al cliente. Este trabajo propone herramientas de mejora logística en cada una de las áreas de oportunidad que conlleven a disminuir costos logísticos, pero no es un modelo integral.

- El modelo de gestión logística propuesto por Velásquez (2003) identifica, en un primer ciclo, la producción, las ventas y la logística; el segundo ciclo incluye la planeación de materiales, la gestión de inventarios y almacenamiento de materia prima, el plan de compras y la colocación de pedidos al proveedor; mientras que el tercer ciclo tiene como eje el plan de ventas y propiamente la ejecución del mismo. El autor describe un modelo integral de la gestión logística para Pyme; sin embargo, el modelo pierde fuerza de integración al momento de identificar los indicadores que una Pyme debe cumplir, puesto que se centran en el cumplimiento de los mismos de forma aislada.

- La Dirección General de Política de la Pequeña y Mediana Empresa de España (Instituto Pyme, 2007) realizó un modelo de gestión logística derivado del SCOR identificando las siguientes áreas: aprovisionamiento, producción, almacenamiento, transporte y distribución, así como el servicio al cliente. Este modelo está diseñado con las características de la Pyme; sin embargo, el trabajo final de esta investigación fue un Manual de buenas prácticas en logística.

En los modelos expuestos, se hace referencia indirecta a la importancia de los flujos de información interna de la gestión logística, como es el caso del SCOR-model, el cual es considerado un modelo estándar de referencia; sin embargo, está enfocado en los tres primeros niveles y no procura prescribir como cada organización particular debería conducir sus negocios o diseñar sus sistemas o flujos de información (Calderón y Francisco, 2005); por esta razón la interrelación entre cada una de las 
áreas de interés es débil, lo que implica una desintegración total del sistema y un reto cuando se pretende adoptar para mejorar la competitividad de una Pyme.

De acuerdo con las necesidades de la Pyme, expuesta en la literatura revisada, se diseñó un modelo conceptual de gestión logística para dichas empresa, el cual podría dar solución integral a través del control de las variables que mejoran la gestión logística de la Pyme. Para verificar que las variables consideradas en cada una de las dimensiones identificadas son las correctas se utilizó el análisis factorial.

\section{Modelo conceptual}

El siguiente modelo de gestión logística para Pyme está basado en información secundaria, la cual permitió diseñar un modelo conceptual que podría ofrecer una solución integral para la gestión de cuatro de las áreas más importantes para la Pyme: (1) inventarios, (2) almacenamiento, (3) producción y (4) distribución, así como la aplicación de las herramientas que mejorarán el desempeño logístico de la cadena de suministro (figura 1 ).

\section{Figura 1}

Diseño de un modelo conceptual de gestión logística para Pyme

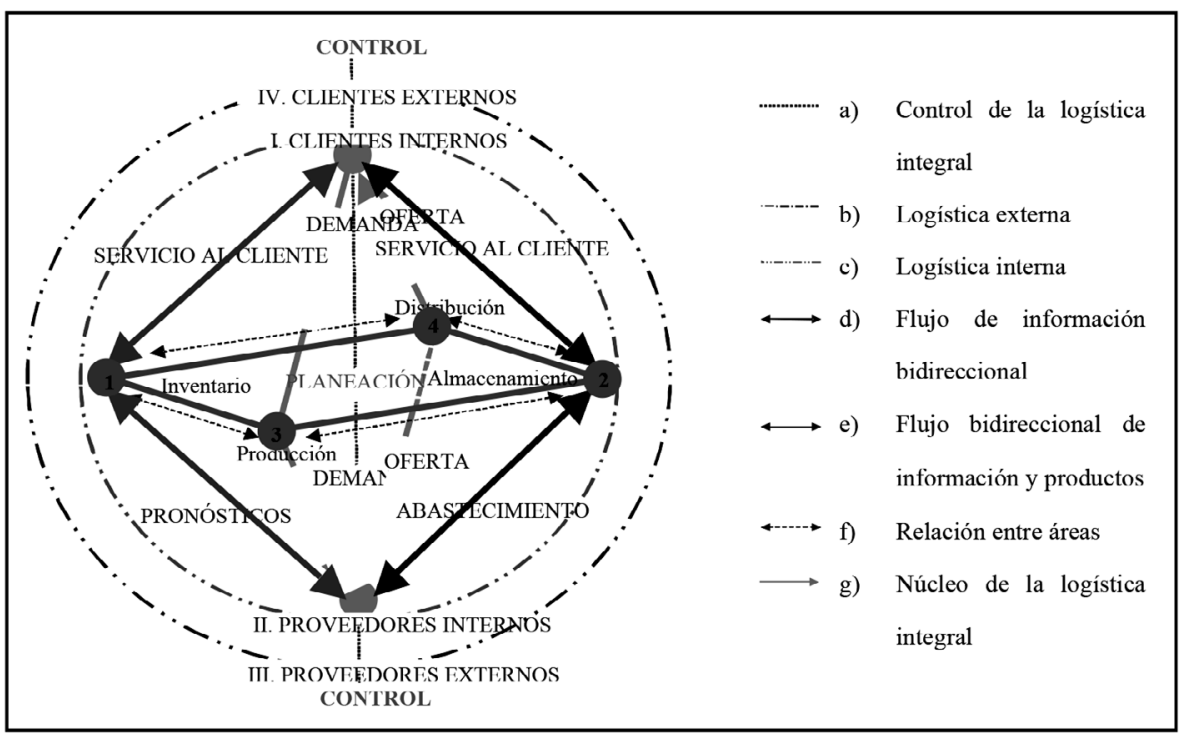


A continuación se muestra el funcionamiento de dicho modelo:

1. Inventarios: el objetivo de esta área es determinar a través de modelos cuantitativos cuánto y cuándo pedir los materiales.

2. Almacenamiento: esta área tiene como objetivo trabajar bajo buenas prácticas para resguardar y controlar eficientemente las materias primas.

3. Producción: su objetivo es trabajar bajo un programa maestro de la producción el cual tendrá que satisfacer los requerimientos del mercado/cliente en tiempo, cantidad y calidad. Es necesario identificar las herramientas necesarias y factibles de mejora continua de aplicación en el proceso de transformación de bienes y del inventario en proceso.

4. Distribución: una alta capacidad de respuesta al cliente al más bajo costo es el objetivo de esta área; esto implica crear valor en la cadena de suministro a través de la optimización del nivel de inventario de producto terminado, así como de tiempo en la transportación y entrega del mismo.

El cliente interno (I) es un miembro que se encuentra en la misma organización que recibe el resultado de un proceso anterior llevado a cabo en la misma organización y que a su vez es proveedor interno (II) del siguiente proceso; aquí es donde se concibe la integración de la logística interna (c).

Los proveedores externos (III) son las empresas que suministran los insumos a la organización para que ésta desarrolle sus negocios; por su parte, el cliente externo (IV) es aquel socio estratégico ajeno a la organización que puede adquirir los bienes de la empresa ya que tiene más opciones para elegir al mejor proveedor. Por lo tanto, la organización debe realizar esfuerzos continuos para captar y fidelizar relaciones a largo plazo con él, pero para que esto suceda también es necesario realizar una exhaustiva evaluación y selección de proveedores externos que garantice el abastecimiento oportuno de insumos de calidad para lograr la satisfacción de los clientes internos y externos. Este proceso da origen a la logística externa (b); sin embargo, para que la logística externa (b) sea eficiente es necesario que primero funcione la logística interna (c).

El núcleo del modelo es el flujo sincronizado de información de clientes de la empresa a proveedores y viceversa (g); es decir, la demanda que da inicio en el nodo superior del octaedro (clientes externos) y la oferta en el nodo inferior (proveedores externos) del mismo. Los flujos de información y productos (d y e) a partir del núcleo $(\mathrm{g})$ generan la demanda pronosticada a través de las ventas históricas y el 
abastecimiento de los materiales necesarios en el tiempo y lugar indicado con un enfoque justo a tiempo; éstos a su vez son el soporte para dar inicio a la planeación de las cuatro áreas principales del modelo $(1,2,3,4)$, que, al mismo tiempo, se interrelacionarán (f), planearán y controlarán (a) como una unidad para que impacten directamente en el nivel de servicio al cliente de la empresa mediante los flujos de información y productos ( $\mathrm{d}$ y e) del sistema. La principal característica del modelo es que debe ser lo suficientemente capaz de compartir la información clave entre todos los miembros de la cadena de suministro (de proveedores internos/externos a clientes internos/externos) para reducir la incertidumbre de la misma, puesto que las decisiones que se tomen en cualquier vértice del octaedro tendrán impacto en todos los demás.

Un sistema híbrido de control de producción empujar/jalar que opera de forma adecuada y continua combinando las ventajas de ambos sistemas puede alcanzar un mayor grado de efectividad. Los sistemas de empujar se definen comúnmente con el pronóstico de la demanda para la planificación de los materiales. Los sistemas jalar son aquellos en los que las cantidades de pedido se determinan sobre la base de la demanda real (Hirakawa et al., 1992).

El modelo trabajará bajo el enfoque de un sistema híbrido empujar/jalar de la cadena de suministro: el proceso de empujar en la cadena se realiza con anticipación a la demanda del cliente con base en un pronóstico de ventas; es decir, se debe planear y controlar el nivel del inventario (1) y de la función de almacenamiento (2).

El proceso de jalar en la cadena se realiza en respuesta a la demanda real del cliente; se debe planear y controlar el nivel de actividad en producción (3) y en la distribución (4).

El sistema de empujar/jalar debe satisfacer al cliente en tiempo, lugar, calidad, cantidad, servicio y con el menor costo posible.

Las dimensiones o factores que se han identificado para dar respuesta a la estructura del modelo conceptual de gestión logística para Pyme son: compras, abastecimiento de materiales, producción, distribución, servicio al cliente y, por último, flujo de información (anexo 1). Cada una de estas dimensiones está conformada por variables (preguntas de la encuesta), mismas que se verifican si son las correctas mediante el análisis factorial. 


\section{Metodología}

Debido al objetivo de la investigación y al tamaño de muestra disponible se realizó un análisis factorial, el cual examina toda la rama de relaciones de interdependencia de las variables que están correlacionadas (Hair et al, 1995).

Se recolectó información primaria a través de encuestas aplicadas en el estado de Tlaxcala debido a los recursos disponibles y cercanía con las instituciones de educación superior donde están adscritos los investigadores. Se eligió el sector textil porque es el más grande, el que aporta más PIB y el que genera más empleos de la industria manufacturera en el nivel estatal (INEGI, 2012). Fueron contactadas 66 pequeñas y medianas empresas de este sector, lo cual corresponde al 100\% de esta población, según el directorio industrial 2012 de la Secretaría de Desarrollo Económico de Tlaxcala. Posteriormente, se realizó un muestreo por conveniencia, pues sólo se encuestaron a empresas que aceptaron participar en esta investigación; la encuesta fue aplicada a personal de mandos altos e intermedios; además, se aplicó una encuesta por empresa. Por consiguiente, el tamaño de muestra fue de 33 encuestas, una por cada empresa, lo cual equivale al $50 \%$ de la población del sector textil de Tlaxcala; estos datos fueron obtenidos durante el periodo febrero-abril de 2013 y analizados a través de las herramientas del software SPSS versión 19.0 para Windows.

El instrumento de medición de esta investigación fue una encuesta que consta de tres secciones; las dos primeras partes se orientan en analizar el perfil del encuestado y la participación de la Pyme del sector textil en el mercado; la tercera sección está conformada por los cinco factores antes mencionados de los cuales se plantearon 21 preguntas (variables). Se utilizó una escala de Likert de 5 opciones/alternativas, la cual va desde totalmente en desacuerdo hasta totalmente de acuerdo (anexo 1). En este documento no se muestra el detalle de las dos primeras secciones.

a) Validación del instrumento de medición. En el instrumento de medición que se aplicó en el estudio y de acuerdo con el criterio de Cronbach (1951) existe alta confiabilidad (cuadro 1). 


\section{Cuadro 1 \\ Estadístico de fiabilidad}

\begin{tabular}{c|c}
\hline Alfa de Cronbach & N de elementos \\
\hline .955 & 21 \\
\hline
\end{tabular}

b) Análisis factorial. Asegura que la matriz de datos tiene suficientes correlaciones para justificar la aplicación del análisis de factores. Hay indicadores estadísticos formales que permiten verificar que el modelo factorial es apropiado (cuadro 2).

\section{Cuadro 2}

\section{Estadísticos para verificar modelo factorial}

\begin{tabular}{l|r}
\hline \multicolumn{2}{c}{ KMO y prueba de Bartlett } \\
\hline Medida de adecuación muestral de Kaiser-Meyer-Olkin. & GI \\
Prueba de esfericidad de Bartlett Chi-cuadrado aproximado & Sig. \\
& 698.149 \\
\hline & 210 \\
\hline
\end{tabular}

En este trabajo, el determinante de la matriz de correlación fue 0.000 , la prueba de Bartlett $\left(X^{2}\right)$ de 698.149 y el KMO de 0.742. Bajo el criterio de Mahlotra (2004) se concluye que los resultados son satisfactorios; por lo tanto, es correcto continuar con el análisis factorial.

Esta investigación tiene 21 variables por analizar, las cuales corresponden a cada una de las preguntas de la encuesta y un total de 33 encuestas, por lo que se tiene una relación de 1.57 encuestas por cada variable.

c) Determinación del número de factores a extraer. Para extraer los factores puede utilizarse el método de análisis de componentes principales debido a que el objetivo es condensar la mayoría de la información original (varianzas) en un número de factores para propósitos de predicción. Los factores por extraer son aquellos que tengan autovalores (eigenvalues) mayores a 1.

Para el desarrollo del estudio se analizaron 21 variables que fueron utilizadas para extraer el número de factores mediante el criterio de la raíz latente (eigenvalues 
<1). La extracción produjo cuatro factores que explican el $78.45 \%$ de la varianza de los datos originales; los demás fueron ignorados por ser no significativos, ya que los factores por extraer son aquellos que tengan valores propios (eigenvalues) mayores a 1 (cuadro 3).

\section{Cuadro 3}

Varianza total explicada

\begin{tabular}{|c|c|c|c|c|c|}
\hline \multirow{2}{*}{ Componente } & \multicolumn{3}{|c|}{ Valores propios iniciales } & \multirow{2}{*}{$\begin{array}{c}\begin{array}{c}\text { Sumas de las } \\
\text { saturaciones al } \\
\text { cuadrado de la } \\
\text { extracción }\end{array} \\
\% \\
\text { acumulado } \\
\end{array}$} & \multirow{2}{*}{\begin{tabular}{|c}
$\begin{array}{c}\text { Suma de las saturaciones } \\
\text { al cuadrado de la } \\
\text { rotación }\end{array}$ \\
$\%$ \\
acumulado \\
\end{tabular}} \\
\hline & Total & $\begin{array}{c}\% \text { de la } \\
\text { varianza }\end{array}$ & $\begin{array}{c}\% \\
\text { acumulado }\end{array}$ & & \\
\hline 1 & 11.433 & 54.442 & 54.442 & 54.442 & 32.994 \\
\hline 2 & 2.644 & 12.592 & 67.035 & 67.035 & 61.410 \\
\hline 3 & 1.375 & 6.547 & 73.582 & 73.582 & 70.638 \\
\hline 4 & 1.023 & 4.870 & 78.452 & 78.452 & 78.452 \\
\hline 5 & 0.936 & 4.458 & 82.910 & & \\
\hline . & . & . & . & & \\
\hline . & . & . & . & & \\
\hline 21 & 0.012 & 0.058 & 100.000 & & \\
\hline
\end{tabular}

d) Rotación de factores. Comrey (1973) indica que los niveles carga de los factores arriba de 0.45 se consideran válidos, arriba de 0.55 se consideran buenos, arriba de 0.63 se consideran muy buenos y arriba de 0.71 son excelentes.

Es necesario emplear un método rotacional para lograr soluciones de factor significativas y más simples. Para la rotación de factores se utilizó el método rotacional ortogonal VARIMAX, ya que se debería reducir el número de variables a un conjunto más pequeño de variables no correlacionadas, y que corresponden a las funciones estratégicas y actividades clave del modelo conceptual, la rotación convergió en seis interacciones (cuadro 4).

Se puede observar en el cuadro 2 que el factor 1 está compuesto por 11 variables; el factor 2 por 7 variables, el factor 3 por 2 variables y el factor 4 por 1 variable; este último se decide eliminar por tener sólo una, por lo que la variable 9 pasa al factor 2 por la afinidad de la variable al factor. Para confirmar la decisión se realizó 
nuevamente la extracción y rotación del análisis factorial forzando este análisis a tres factores (cuadro 5), donde la variable 9 se reagrupó en el factor 2.

Cuadro 4

Matriz de componentes rotados ${ }^{\mathrm{a}}$

\begin{tabular}{|c|c|c|c|c|}
\hline \multirow{2}{*}{ Variable } & \multicolumn{4}{|c|}{ Componente } \\
\cline { 2 - 5 } & $\mathbf{1}$ & $\mathbf{2}$ & $\mathbf{3}$ & $\mathbf{4}$ \\
\hline VAR00015 & 0.792 & & & \\
VAR00003 & 0.778 & & & \\
VAR00020 & 0.769 & & & \\
VAR00019 & 0.768 & & & \\
VAR00018 & 0.765 & & & \\
VAR00004 & 0.765 & & & \\
VAR00017 & 0.747 & & & \\
VAR00007 & 0.728 & & & \\
VAR00005 & 0.714 & 0.512 & & \\
VAR00021 & 0.690 & & & \\
VAR00006 & 0.681 & 0.483 & & \\
VAR00011 & & 0.875 & & \\
VAR00013 & & 0.837 & & \\
VAR00010 & & 0.812 & & \\
VAR00008 & & 0.811 & & \\
VAR00012 & & 0.788 & & \\
VAR00016 & 0.534 & 0.682 & & \\
VAR00014 & & 0.619 & & \\
VAR00002 & & & & \\
VAR00001 & & 0.575 & 0.854 & \\
VAR00009 & & 0.531 & 0.684 & \\
& & & & \\
\hline
\end{tabular}

Cuadro 5 Matriz de componentes rotados ${ }^{\mathrm{a}}$

\begin{tabular}{|c|c|c|c|}
\hline \multirow{2}{*}{ Variable } & \multicolumn{3}{|c|}{ Componente } \\
\cline { 2 - 4 } & $\mathbf{1}$ & $\mathbf{2}$ & $\mathbf{3}$ \\
\hline VAR00021 & 0.826 & & \\
VAR00019 & 0.819 & & \\
VAR00004 & 0.795 & & \\
VAR00020 & 0.793 & & \\
VAR00018 & 0.772 & & \\
VAR00015 & 0.749 & & \\
VAR00007 & 0.723 & & \\
VAR00003 & 0.723 & & \\
VAR00017 & 0.704 & & \\
VAR00006 & 0.677 & 0.520 & \\
VAR00005 & 0.658 & 0.539 & \\
VAR00011 & & 0.890 & \\
VAR00013 & & 0.851 & \\
VAR00010 & & 0.834 & \\
VAR00008 & & 0.822 & \\
VAR00012 & & 0.812 & \\
VAR00016 & 0.509 & 0.711 & \\
VAR00014 & & 0.669 & \\
VAR00009 & & 0.587 & \\
VAR00002 & & & \\
VAR00001 & & 0.592 & \\
& & & \\
\hline
\end{tabular}

Así, los tres factores independientes se redefinen y se conforman como se señala en el cuadro 6; se puede observar que las mediciones de las preguntas de la encuesta (anexo 1) muestran un alto grado de agrupamiento en cada uno de los factores. 


\section{Cuadro 6}

Agrupación de variables

\begin{tabular}{|c|c|c|c|c|}
\hline Área & $\begin{array}{l}\text { Pregunta } \\
\text { de la } \\
\text { encuesta }\end{array}$ & Factor & $\begin{array}{c}\text { Carga } \\
\text { de la } \\
\text { variable }\end{array}$ & $\begin{array}{l}\text { Redefiniendo el } \\
\text { nuevo factor }\end{array}$ \\
\hline \multirow{4}{*}{ Compras } & 1 & 3 & 0.660 & \multirow{2}{*}{$\begin{array}{c}\text { Administración de } \\
\text { la demanda }\end{array}$} \\
\hline & 2 & 3 & 0.853 & \\
\hline & 3 & 1 & 0.723 & \multirow{6}{*}{$\begin{array}{l}\text { Administración de } \\
\text { abastecimiento y } \\
\text { distribución }\end{array}$} \\
\hline & 4 & 1 & 0.795 & \\
\hline \multirow{2}{*}{$\begin{array}{c}\text { Abastecimiento de } \\
\text { materiales }\end{array}$} & 5 & 1 & 0.658 & \\
\hline & 6 & 1 & 0.677 & \\
\hline \multirow{10}{*}{ Producción } & 7 & 1 & 0.723 & \\
\hline & 15 & 1 & 0.749 & \\
\hline & 8 & 2 & 0.822 & \multirow{8}{*}{$\begin{array}{l}\text { Administración de } \\
\text { la producción }\end{array}$} \\
\hline & 10 & 2 & 0.834 & \\
\hline & 11 & 2 & 0.890 & \\
\hline & 12 & 2 & 0.812 & \\
\hline & 13 & 2 & 0.851 & \\
\hline & 14 & 2 & 0.669 & \\
\hline & 16 & 2 & 0.711 & \\
\hline & 9 & 2 & 0.587 & \\
\hline \multirow{2}{*}{$\begin{array}{c}\text { Distribución y } \\
\text { servicio al cliente }\end{array}$} & 17 & 1 & 0.704 & \multirow{5}{*}{$\begin{array}{l}\text { Administración de } \\
\text { abastecimiento y } \\
\text { distribución }\end{array}$} \\
\hline & 18 & 1 & 0.772 & \\
\hline \multirow{3}{*}{ Flujo de información } & 19 & 1 & 0.819 & \\
\hline & 20 & 1 & 0.793 & \\
\hline & 21 & 1 & 0.826 & \\
\hline
\end{tabular}

A manera de comprobación de los resultados del análisis factorial, en éste se utilizó el método split-half dando como resultado un alfa de Cronbach alto, tanto en la parte uno como en la parte dos (cuadro 7); de esta manera, el análisis factorial realizado procede. 


\section{Cuadro 7 \\ Método split-half}

\begin{tabular}{|c|c|c|c|}
\hline \multicolumn{4}{|c|}{ Estadísticos de fiabilidad } \\
\hline \multirow{5}{*}{ Alfa de Cronbach } & \multirow{2}{*}{ Parte 1} & Valor & 0.911 \\
\hline & & $\mathrm{N}$ de elementos & $11^{2}$ \\
\hline & \multirow{2}{*}{ Parte 2} & Valor & 0.917 \\
\hline & & $\mathrm{N}$ de elementos & 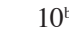 \\
\hline & \multicolumn{2}{|c|}{$\mathrm{N}$ total de elementos } & 21 \\
\hline \multicolumn{3}{|l|}{ Correlación entre formas } & 0.908 \\
\hline \multirow[t]{2}{*}{ Coeficiente de Spearman-Brown } & & itud igual & 0.952 \\
\hline & & ad desigual & 0.952 \\
\hline \multicolumn{4}{|l|}{ Dos mitades de Guttman } \\
\hline \multicolumn{3}{|c|}{ a. Los elementos son las variables: $01,02,03,04,05,06,07,08,09,10,11$} & \\
\hline \multicolumn{3}{|c|}{ b. Los elementos son las variables: $11,12,13,14,15,16,17,18,19,20,21$} & 0.952 \\
\hline
\end{tabular}

Una vez concluido el análisis factorial se validó la confiabilidad de las mediciones resultantes a través de los valores del alfa de Cronbach, los cuales se encontraron aceptables; de esta manera se demuestra que las mediciones propuestas tienen un alto nivel de congruencia interna (cuadro 8).

\section{Cuadro 8}

Pruebas de confiabilidad de alfa de Cronbach

\begin{tabular}{c|c|c}
\hline Factor & $\begin{array}{c}\text { No. de } \\
\text { elementos }\end{array}$ & $\begin{array}{c}\text { Alfa de } \\
\text { Cronbach }\end{array}$ \\
\hline Administración de abastecimiento y & 11 & 0.945 \\
distribución & 8 & 0.939 \\
Administración de la producción & 2 & 0.850 \\
\hline Administración de la demanda & & \\
\hline
\end{tabular}

\section{Modelo resultante}

Una vez concluido el análisis de la información se hace evidente que las variables por controlar en el diseño del modelo conceptual de gestión logística para Pyme son las correctas debido a que no hubo pérdida de información, sólo se muestra una redefinición de factores (figura 2), por lo que se considera que este modelo podría contribuir a resolver de forma integral y estratégica las necesidades logísticas de la Pyme. 
Figura 2

\section{Modelo conceptual de gestión logística para Pyme}

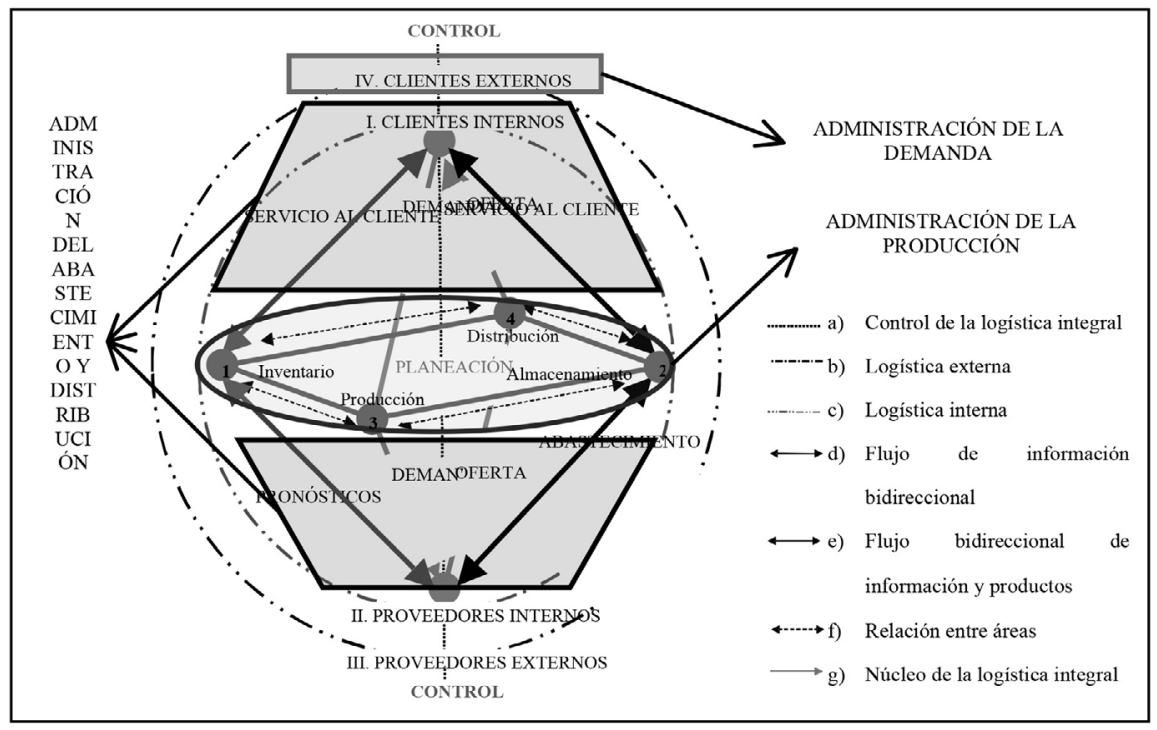

El factor Administración del abastecimiento y distribución debe integrar las áreas y funciones, dentro y fuera de la empresa, mediante el suministro de la información del sistema logístico; de la misma forma debe desempeñar eficientemente el suministro de los materiales y la entrega de los pedidos al cliente externo en tiempo, lugar, calidad, cantidad, servicio y al menor costo posible; es decir, es necesario coordinar las áreas estratégicas a través de la sincronización de las funciones de los tres factores resultantes para fortalecer el sistema híbrido de producción empujar/jalar.

Para asegurar el buen desempeño entre los factores Administración de abastecimiento y distribución con la Administración de la producción se debe controlar el tiempo de ciclo de la orden de compra y estandarizar el surtimiento de materiales a las estaciones de trabajo en tiempo, secuencia y cantidad a través de modelos de inventarios.

De la misma forma, con el factor Administración de la demanda se debe considerar el comportamiento histórico de las ventas para realizar las estimaciones o pronósticos de la demanda a través de métodos cuantitativos y, a su vez, controlar el tiempo 
de entrega de los pedidos de los clientes y la eficacia de los despachos efectuados teniendo en cuenta pedidos completos a tiempo con documentación perfecta y sin daños a la mercancía.

Mediante los flujos de información se interrelacionan las cuatro áreas del factor Administración de la producción de tal manera que se facilita en cada una de ellas la aplicación eficaz de las herramientas de mejora continua (SMED, 5's, herramientas visuales, sistema de flujo continuo, eliminación de los siete desperdicios, manufactura esbelta, entre otros); mismas que permiten dar cumplimiento a los requerimientos de calidad que los clientes exigen en los productos.

\section{Conclusiones}

En este artículo se presentó el diseño de un modelo de gestión logística para Pyme; además, se verificó que las variables por controlar en cada proceso logístico son las correctas. Por la simplicidad del mismo es factible que este tipo de empresas lo adopten como referencia para mejorar el desempeño logístico de la cadena de suministro. Es importante que en cada área se aproveche el conocimiento técnico, la experiencia, la información de clientes internos y externos para desarrollar, diferenciar y diseñar nuevos productos en los mercados globalizados. La Pyme también puede formar parte de una red de clientes y proveedores vinculados a grandes empresas en tanto entiendan el funcionamiento de la nueva dinámica de trabajar en el esquema de "cadena global de insumos" y en el esquema de "paquete completo" para ciertos productos.

Tomar este modelo como referencia podría permitir a los gestores de pequeñas y medianas empresas en México la mejora en el desempeño logístico de la cadena de suministro, así como mejorar su competitividad y enfrentar los retos de mercados nacionales e internacionales.

Las empresas que tienen un buen desempeño logístico y que logran una ventaja competitiva están proporcionando un nivel de servicio superior a sus clientes, aunque es desafiante integrar las combinaciones de recursos, habilidades y sistemas requeridos para lograr una logística adecuada, pero si esto se alcanza, difícilmente los competidores duplicarán tal desempeño integrado. 
Algunas limitaciones que se perciben en este estudio son, por una parte, la cultura empresarial de no trabajar con buenas prácticas; y, por otro, la resistencia al cambio y a colaborar en la implementación de una reingeniería de sus procesos logísticos.

En futuras investigaciones se recomiendan las siguientes líneas de investigación.

1. Implementar el modelo en alguna Pyme manufacturera para evaluar su pertinencia, pudiendo ser en una textil de la región Puebla-Tlaxcala o de cualquier otro sector y región.

2. Evaluar cada uno de los actores y variables que intervienen en los procesos internos (procesos culturales, capacitación, talento humano, tecnologías de información, sistemas de inteligencia de mercado, sistemas de información gerencial) que pudieran incrementar la eficiencia en la producción, en la administración de la cadena global de suministro.

3. Transportar, implementar y evaluar el modelo en otros sectores productivos y regiones.

\section{Referencias}

Ballou, R. H. (2004). Logística: administración de la cadena de suministro. $5^{\mathrm{a}}$ ed. México: Prentice Hall.

Banco Interamericano de Desarrollo (2011). La logística como factor de competitividad de las Pymes en las Américas. Disponible en: http://idbdocs.iadb.org/ wsdocs/get document.aspx?docnum=36610289

Banco Mundial (2012). Compete 2012. Disponible en: http://web.worldbank. org/ WBSITE/EXTERNAL/TOPICS/DE/0, contentMDK:23188613 pagePK:210058 piPK:210062 theSitePK:239071,00.html

Calderón, J. L. y E. L. Francisco (2005). Análisis del modelo SCOR para la gestión de la cadena de suministro, Ponencia, IX Congreso de Ingeniería de Organización, Gijón, España.

Comrey, A. (1973). A first course in factor analysis. $2^{\mathrm{a}}$ ed. Nueva York: Academic Press.

Consejo Ejecutivo de Empresas Globales (2004). Reflexiones sobre la competitividad en México. Disponible en : http://www.empresasglobales.org.mx/ pdfAdmin/70refle xiones.pdf 
Cronbach, L. (1951). Coefficient alpha and the internal structure of tests. Psychometrica 16 (3): 297-334.

Daft, R. L. (2011). Teoría y diseño organizacional. 10ª ed. México: Cengage Learning.

Díaz, G. H., M. A. Corona y Y. Mayett. (2012). Los retos de las empresas familiares ante su continuidad: caso Tehuacán. Revista Internacional Administración y Finanzas 6 (1): 1-22.

Díaz, H. B., R. G. García y N. Porcell (2008). Las Pymes: costos en la cadena de abastecimiento. Revista Escuela de Administración de Negocios, mayo-agosto 63: 5-22.

Domínguez, R. M. C. (2010). El programa de competitividad e innovación tecnológica (COMPITE) en la industria de la confección textil. Empresa textil y de la confección: trayectorias, alianzas y apoyos a la innovación. México: Benemérita Universidad Autónoma de Puebla: 29-49.

Gobierno Federal (2007). Plan Nacional de Desarrollo 2007-2012. Disponible en: http://pnd.calderon.presidencia.gob.mx/pdf/PrimerInformeEjecucion/2_6. pdf

González, C. A., J. L. Martínez, C. Malcón y J. Cavazos (2012). Metodología de gestión logística para el mejoramiento de pequeñas empresas. Global Conference on Business and Finance Proceedings 7 (2): 1007-1015.

Hair, J. F., R. E. Anderson, R. L. Tatham y W. C. Black (1995). Multivariate data analysis with readings. 4 a ed. Nueva Jersey: Prentice Hall.

Hernández-Laos, E. (2000). La competitividad industrial en México. México: Plaza y Valdés.

Hirakawa, Y., K. Hoshino y H. Katayama (1992). A hybrid push/pull production control system for multistage manufacturing processes. International Journal of Operations and Production Management 12 (4): 69-81. 
Instituto Mexicano para la Competitividad (2010). Competitividad urbana 2010. Disponible en: http://imco.org.mx/ciudades2010/librocompleto.pdf

Instituto Nacional de Estadística Geografía e Informática (INEGI) (2011). Micro, pequeña, mediana y gran empresa. Estratificación de los establecimientos. Censos económicos 2009. Disponible en: http://www.inegi.org.mx/est/contenidos/ espanol/proyectos/censos/ce2009/pdf/Mono_Micro_peque_mediana.pdf

(2012). Anuario estadístico de Tlaxcala 2012. Serie anual 2007-2011. Disponible en: http://www.inegi.org.mx/prod_serv/contenidos/espanol/bvinegi/productos/integracion/pais/anuario_multi/2012/tlax/702825046163. pdf

Instituto PYME (2007). Logística y competitividad de las Pyme. Disponible en: http://www.ipyme.org

Instituto Tecnológico Autónomo de México (2010). La industria textil en México. Diagnóstico, prospectiva y estrategia. Disponible en: http://cec.itam.mx/ medios_digitales/documentos/Estudios_sectoriales/Resumenes_Ejecutivos/ Textil.pdf

Mahlotra, N. (2004). Investigación de mercados. Un enfoque aplicado. $4^{\mathrm{a}}$ ed. México: Pearson Prentice-Hall.

Robles, G. y J. Carlos. (2003). Administración: un enfoque interdisciplinario. México: Pearson Educación.

Rodríguez, J. (2003). Administración de pequeñas y medianas empresas. 5 ed. México: Cengage Learning Editores.

Seccretaría de Desarrollo Económico (2012). Directorio industrial del estado de Tlaxcala. Disponible en: http://www.sedecotlaxcala.gob.mx/index2/images/archivos/directorio/dir_oct2011.pdf

Secretaría de Economía (2011). Políticas públicas para el desarrollo de la logística y la gestión de la cadena de suministro en México. Disponible en: http:// www.mecon.gov.ar/peconomica/dnper/documentos/MX_RHernandez_Politicas_Publicas.pdf 
Supply Chain Council (1996). Supply-chain operations reference-model. Disponible en: http://supply-chain.org/f/SCOR-Overview-Web.pdf

Velásquez, A. (2003). Modelo de gestión de operaciones para Pymes innovadoras. Escuela de Administración de Negocios, enero-abril (47): 66-87

Vera, M, G. S. (2010). La industria textil en México. En M. C. Domínguez Ríos. Empresa textil y de la confección: trayectorias, alianzas y apoyos a la innovación. México: Benemérita Universidad Autónoma de Puebla: 13-28.

\section{Anexo 1 \\ Instrumento para medir el desempeño logístico de Pymes textiles}

\begin{tabular}{|c|c|}
\hline \multicolumn{2}{|l|}{ CUESTIONARIO } \\
\hline \multicolumn{2}{|c|}{$\begin{array}{l}\text { Objetivo: Realizar un diagnóstico del desempeño logístico de las PYMES del sector textil, que permita } \\
\text { proponer un Modelo de Gestión Logística para aumentar la competitividad en el sector. }\end{array}$} \\
\hline \multicolumn{2}{|c|}{$\begin{array}{l}\text { Agradecemos su participación en este ejercicio y el esfuerzo en reportar los datos con alta confiabilidad. La } \\
\text { información que se reciba se tratará con total confidencialidad ya que el estudio es estrictamente académico. }\end{array}$} \\
\hline \multicolumn{2}{|c|}{$\begin{array}{l}\text { Instrucciones: Lea cuidadosamente todo el documento y marque con una } X \text { dentro del circulo la respuesta } \\
\text { seleccionada (1. Totalmente en desacuerdo } 2 \text {. En desacuerdo } 3 \text {. Indeciso } 4 \text {. De acuerdo } 5 \text {. Totalmente de acuerdo) }\end{array}$} \\
\hline \multicolumn{2}{|l|}{ COMPRAS } \\
\hline $\begin{array}{l}\text { El análisis del comportamiento histórico de las ventas es parte esencial y regular del } \\
\text { proceso de estimación de la demanda. }\end{array}$ & (1) (2) (3) (4) (5) \\
\hline $\begin{array}{l}\text { Se realizan las estimaciones o pronósticos de la demanda a través de métodos } \\
\text { cuantitativos. }\end{array}$ & (1) (2) (3) (4) (5) \\
\hline $\begin{array}{l}\text { Se mide o monitorea el tiempo desde que la orden de compra ha sido entregada al } \\
\text { proveedor hasta que el producto es recibido en el Almacén. }\end{array}$ & (1) (2) (3) (4) (5) \\
\hline $\begin{array}{l}\text { Al colocar órdenes de compra al proveedor se considera el costo de ordenar (papeleo, } \\
\text { administración y procesamiento de la orden, transporte, recibo e inspección de la } \\
\text { mercancía, contabilidad, etc.) y el costo de mantener inventario (costo de oportunidad, } \\
\text { riesgo de obsolescencia, daños, seguro, espacio y manejo, entre otros). }\end{array}$ & (1) (2) (3) (4) (5) \\
\hline \multicolumn{2}{|l|}{$\begin{array}{l}\text { ABASTECIMIENTO DE MATERIALES } \\
\end{array}$} \\
\hline $\begin{array}{l}\text { El proceso de surtimiento de materiales a las estaciones de trabajo es calculado, medido } \\
\text { y mejorado constantemente. }\end{array}$ & (1) (2) (3) (4) (5) \\
\hline $\begin{array}{l}\text { El surtimiento de materiales a las estaciones de trabajo está estandarizado en cuanto a } \\
\text { tiempo, secuencia y cantidad. }\end{array}$ & (1) (2) (3) (4) (5) \\
\hline \multicolumn{2}{|l|}{ PRODUCCIÓN } \\
\hline $\begin{array}{l}\text { Están definidas y delimitadas las ubicaciones de los materiales en las estaciones de } \\
\text { trabajo para facilitar las tareas a los operadores. }\end{array}$ & (1) (2) (3) (4) (5) \\
\hline
\end{tabular}




\begin{tabular}{|c|c|}
\hline $\begin{array}{l}\text { La programación de la producción es determinada por las órdenes enviadas por el } \\
\text { cliente. }\end{array}$ & (1) (2) (3) (4) (5) \\
\hline $\begin{array}{l}\text { Las estaciones de trabajo cuentan con herramientas visuales que muestren el programa } \\
\text { de producción. }\end{array}$ & (1) (2) (3) (4) (5) \\
\hline $\begin{array}{l}\text { Los cambios de herramental se realizan en un tiempo breve para permitir programar } \\
\text { cambios de versión de productos en el periodo de producción. }\end{array}$ & (1) (2) (3) (4) (5) \\
\hline Los métodos de trabajo están especificados en todos los niveles de la organización. & (1) (2) (3) (4) (5) \\
\hline Existe un sistema de orden y limpieza en la organización. & (1) (2) (3) (4) (5) \\
\hline $\begin{array}{l}\text { Las estaciones de trabajo son totalmente flexibles a los cambios solicitados por el } \\
\text { cliente. }\end{array}$ & (1) (2) (3) (4) (5) \\
\hline El proceso de producción trabaja bajo un sistema de flujo continuo. & (1) (2) (3) (4) (5) \\
\hline Se cumplen los requerimientos de calidad que los clientes exigen en los productos. & (1) (2) (3) (4) (5) \\
\hline $\begin{array}{l}\text { Se controla el tiempo desde que la orden de producción se entrega a piso productivo } \\
\text { hasta que llega al almacén de producto terminado. }\end{array}$ & (1) (2) (3) (4) (5) \\
\hline \multicolumn{2}{|l|}{ DISTRIBUCIÓN Y SERVICIO AL CLIENTE } \\
\hline $\begin{array}{l}\text { Se mide y controla el tiempo de entrega de los pedidos de los clientes en un periodo } \\
\text { determinado. }\end{array}$ & (1) (2) (3) (4) (5) \\
\hline $\begin{array}{l}\text { Se mide y controla la eficacia de los despachos efectuados por la empresa teniendo } \\
\text { en cuenta pedidos completos a tiempo, con documentación perfecta y sin daños a la } \\
\text { mercancía. }\end{array}$ & (1) (2) (3) (4) (5) \\
\hline \multicolumn{2}{|l|}{$\begin{array}{l}\text { FLUJO DE INFORMACIÓN } \\
\end{array}$} \\
\hline $\begin{array}{l}\text { La integración de las áreas y sus funciones dentro de la empresa y fuera de ella se } \\
\text { facilita mediante la información en el sistema logístico. }\end{array}$ & (1) (2) (3) (4) (5) \\
\hline Cuenta con la información interna en tiempo y forma que requiere el sistema logístico. & (1) (2) (3) (4) (5) \\
\hline $\begin{array}{l}\text { Hay alguien que administre la información sistematizada y oportuna del entorno y se } \\
\text { las haga llegar. }\end{array}$ & (1) (2) (3) (4) (5) \\
\hline
\end{tabular}


